\title{
Primary Syphilis Presenting As a Chronic Lip Ulcer
}

\author{
Claire Porterfield ${ }^{1}$, David Brodell ${ }^{2}$, Lindsey Dolohanty ${ }^{2}$, Glynis Scott ${ }^{3}$ \\ 1. School of Medicine, University of Rochester, Rochester, USA 2. Dermatology, University of Rochester, Rochester, \\ USA 3. Dermatopathology, University of Rochester, Rochester, USA
}

Corresponding author: Claire Porterfield, claireporterfield1@gmail.com

\begin{abstract}
Syphilis is usually a sexually transmitted infection caused by the spirochete Treponema pallidum. Primary syphilis classically presents as a painless, ulcerated lesion on the genitals. However, the primary lesion is not restricted to this site and appears wherever the spirochete enters through the skin. The symptomatology and appearance of the primary lesion can also vary.
\end{abstract}

We present a case of a 59-year-old man with a primary syphilitic chancre of the lower lip. The patient was referred to the dermatology clinic by their primary care provider after the ulceration failed to heal with antibiotic therapy. A biopsy of the lesion was taken at this time; the diagnosis of syphilis was then made by histologic examination and immunohistochemical staining. Subsequent serologic tests were also positive. Upon prompting, the patient did report a history of sexually transmitted disease but not of syphilis specifically. The patient was treated with penicillin, and there was clinical improvement of the lesion at the follow-up visit.

Received 01/29/2020 Review began 02/20/2020 Review ended 02/23/2020 Published 02/24/2020

(c) Copyright 2020

Porterfield et al. This is an open access article distributed under the terms of the Creative Commons Attribution License CC-BY 4.0., which permits unrestricted use, distribution, and reproduction in any medium, provided the original author and source are credited.
Categories: Dermatology, Family/General Practice, Pathology

Keywords: chronic ulcer, primary syphilis, syphilis, chancre, histology, skin, cutaneous, biopsy, oral syphilis, treponema pallidum

\section{Introduction}

Primary syphilis classically presents as a painless, ulcerated lesion on the genitals. However, it can present in other locations, such as the lips, and with variable appearance, such as painful herpetic-like lesions or vascular pyogenic granuloma-like lesions $[1,2]$. The diagnosis can be made by serologic testing or by histologic examination. Once the diagnosis of syphilis has been established, treatment is usually straightforward; penicillin is the standard treatment and doxycycline is an acceptable alternative in most cases [3].

\section{Case Presentation}

\section{Initial history and presentation}

A 59-year-old male presented to his primary care physician with a two-week history of an excoriated "pimple" on his left chin. The patient denied any previous similar lesions. The patient had a history of asthma, hypertension, depression, arthritis, and chronic back pain. He vaguely recalled a resolved sexually transmitted infection many years before. His only medications were losartan, albuterol, and ibuprofen.

Physical examination revealed a tender $2 \times 3 \mathrm{~cm}$, edematous, hemorrhagic, crusted plaque on the left lower lip that crossed the vermillion border (Figure 1). No purulence was present. The oral mucosa was normal, and there was no lymphadenopathy. The patient was afebrile. 


\section{Cureus}

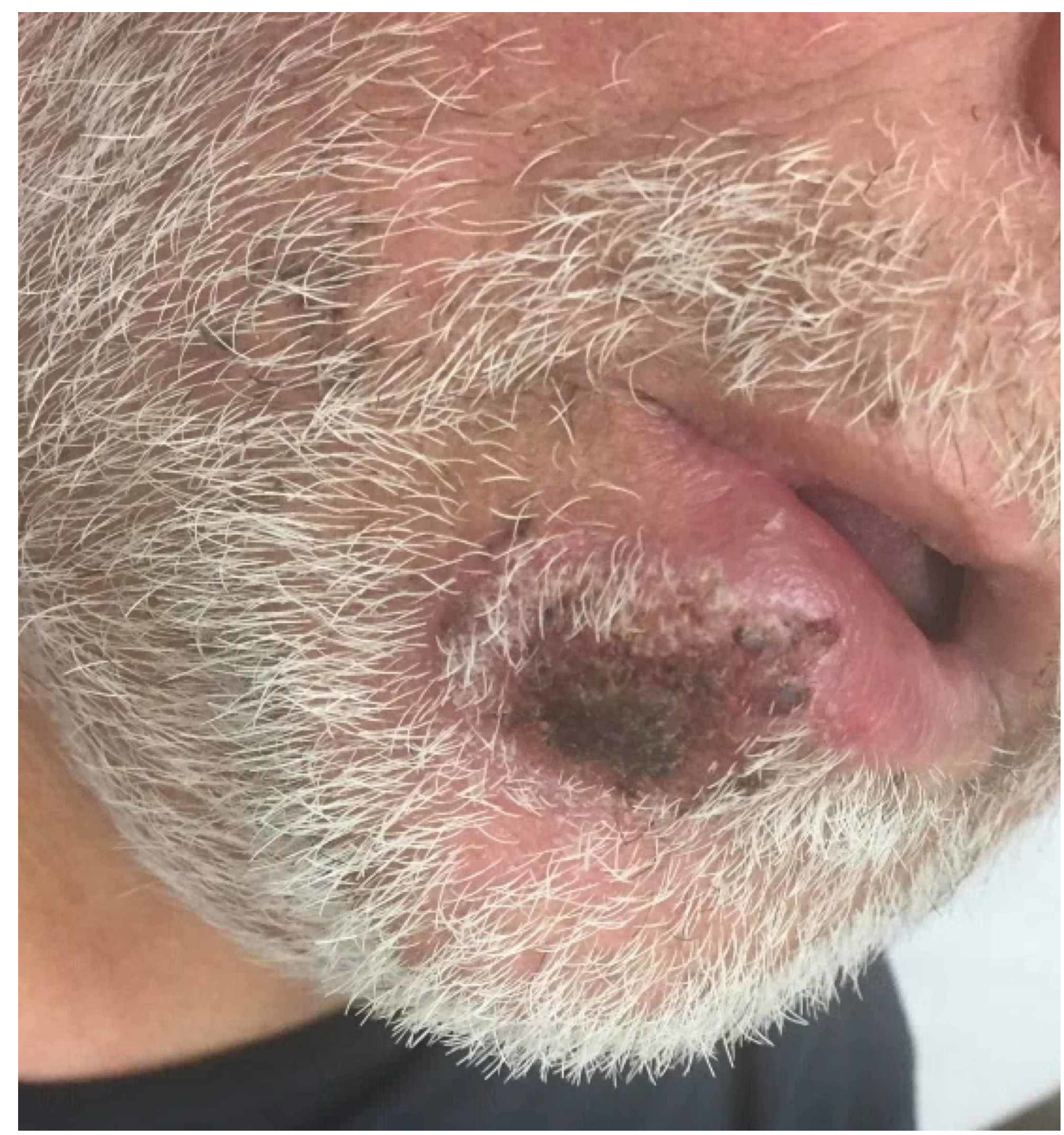

FIGURE 1: Syphilitic chancre presenting as hemorrhagic, crusted plaque on the right cutaneous lip with associated edema.

The lesion did not respond to a course of oral clindamycin prescribed by his primary care provider before seeing dermatology; subsequent incision and drainage revealed a bloody discharge without purulence or signs of gross infection.

\section{Further work-up}

On referral to a dermatology clinic, a punch biopsy was performed given concern for squamous cell carcinoma or deep fungal infection. The biopsy revealed a lichenoid infiltrate associated with numerous plasma cells (Figure 2). The presence of plasma cells prompted immunohistochemical staining for Treponema pallidum, which revealed numerous organisms (Figure 3). A diagnosis of primary syphilis was made. 


\section{Cureus}

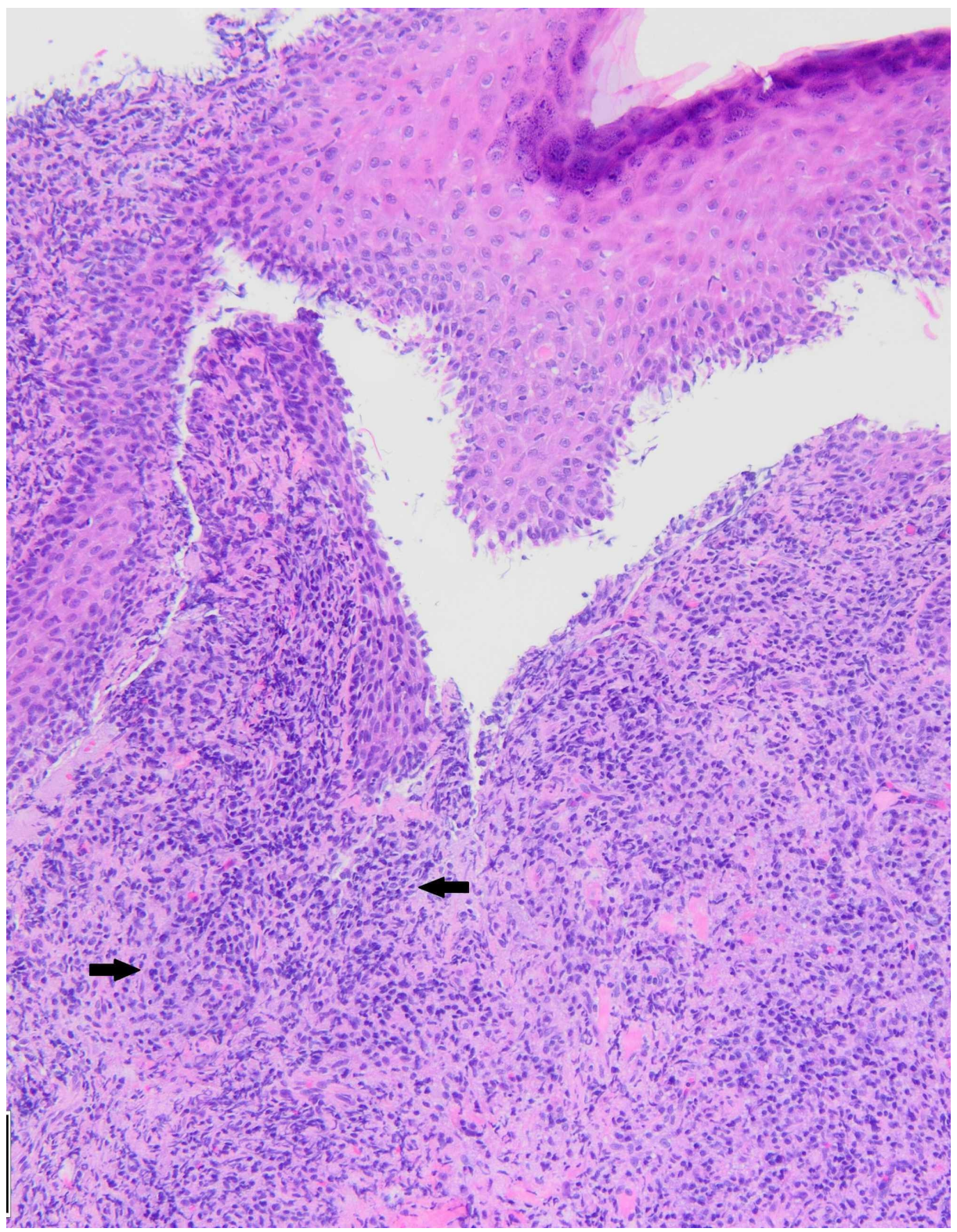

FIGURE 2: Hematoxylin and eosin staining of a skin biopsy at 10x magnification.

The skin is oriented with the surface at the top of the image. There is a diffuse, dense inflammatory cell infiltrate that fills the upper dermis. Within this inflammatory infiltrate are abundant plasma cells (arrows), which are discernible by their hyperchromatic (dark purple) nuclei. 


\section{Cureus}

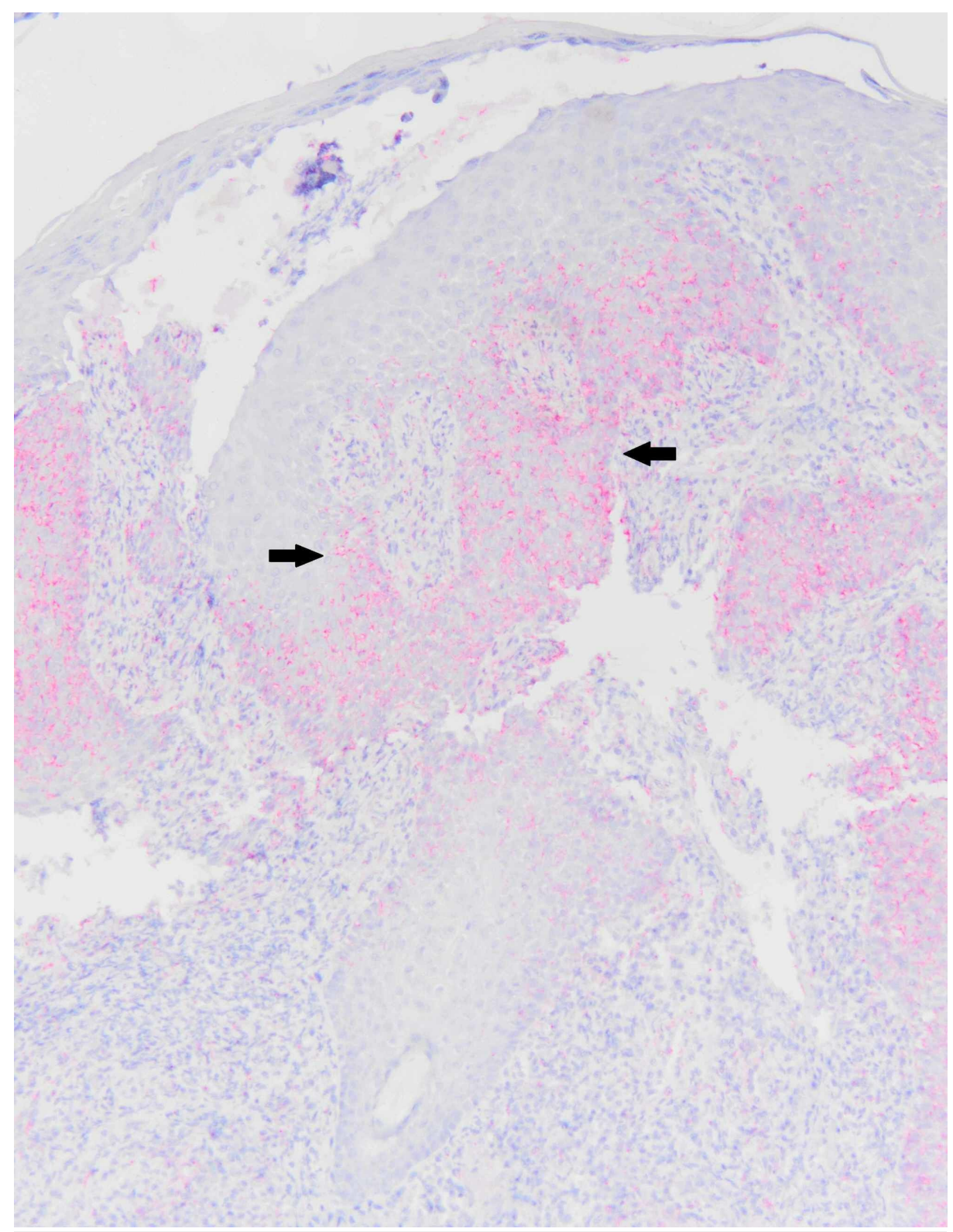

\section{FIGURE 3: Immunohistochemical stain for Treponema pallidum (10x magnification).}

The skin is oriented with the surface at the top of the image. Within the epidermis, the stain highlights thin Treponema pallidum organisms in pink (arrows).

Rapid plasma reagin (RPR) serologic testing and the Treponema pallidum particle agglutination assay were positive, consistent with this diagnosis. Additional screening for human immunodeficiency virus (HIV), Chlamydia trachomatis, Neisseria gonorrhoeae, and hepatitis B and C infection was negative.

\section{Discussion}

\section{Current review of syphilis in the United States}

The incidence of syphilis in the United States has been steadily rising since 2001, with 25,133 cases diagnosed in 2016. Initially, this increase was primarily among men, especially men who have sex with men. However, more recently, increases have been seen in both the male and female population [4].

Syphilis enters the human body through microabrasions in the skin. Approximately 21 days after exposure, a primary syphilitic chancre appears. It is frequently a painless papule with a propensity to ulcerate. Chancres are not associated with an exudate, but regional lymphadenopathy is often present [5]. The great majority of chancres present as genital lesions; however, they can present at other sites, as in this case, with $10 \%$ appearing on the anus, $4 \%$ orally, and $1 \%$ elsewhere [2]. 


\section{Cureus}

Multiple primary chancres can occur, especially in patients co-infected with HIV. The chancre will usually resolve without treatment in a matter of weeks [6]. Primary lesions are highly infectious with a transmission rate of $30 \%$; in contrast, latent lesions have low infectious potential [5]. Oral sex plays an important role in the transmission of syphilis, with around $14 \%$ of patients reporting this as their only risk factor for the disease [7]. When the lesions do present on the genitals, they often do not resemble the classic ulcerated lesion seen in our patient. They can be painful, resembling genital herpes, nodular, or lichen planus-like lesions [1].

\section{Treatment}

The treatment of syphilis is determined by the stage of the disease: early manifestations are treated with penicillin, 2.4 million units once; treatment for late latent or tertiary syphilis is treated weekly and neurosyphilis is treated hourly. Doxycycline can be used as an alternative for penicillin-allergic patients, although this has not been proven efficacious in neurosyphilis or syphilis in pregnant women [3].

The disease becomes more difficult to treat over time; however, treatment early in the patient's disease course (in the primary, secondary, or early latent stages) is usually curative, resulting in disease progression only $10 \%$ of the time [8]. Treatment in the early stages can precipitate a Jarisch-Herxheimer reaction, manifesting as fever, headache, and hypotension, which should not be mistaken for a penicillin allergy. Patients should be monitored serologically for decline in RPR titers at 6 and 12 months. The majority of patients treated in the early stages of disease will serorevert by 36 months [3].

Our patient was treated with a single dose of benzathine penicillin, 2.4 million units. Four weeks after treatment, his primary chancre was dramatically improved (Figure 4).

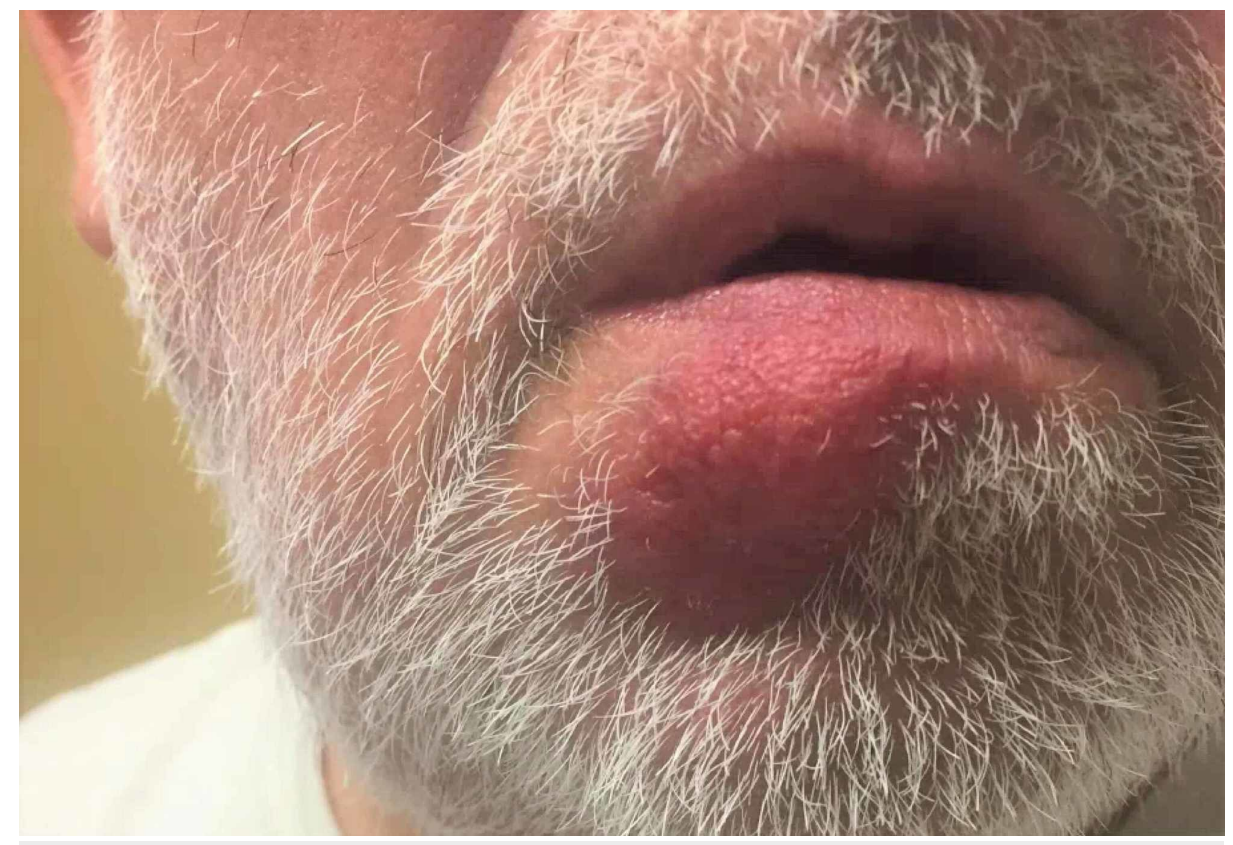

\section{FIGURE 4: Resolving syphilitic chancre of the left cutaneous lip, four weeks after treatment.}

\section{Conclusions}

For any non-healing ulcer, the differential diagnosis is broad, including chancroid, herpes simplex, tuberculous chancre, deep fungal infection, cellulitis, malignancy, traumatic ulcer, and aphthous stomatitis. Our patient highlights the need for a high index of suspicion for syphilitic chancres presenting in nongenital locations. The early diagnosis of primary syphilis is important given its high incidence of transmission and the serious consequences associated with untreated disease.

\section{Additional Information}

\section{Disclosures}

Human subjects: Consent was obtained by all participants in this study. Conflicts of interest: In compliance with the ICMJE uniform disclosure form, all authors declare the following: Payment/services info: All authors have declared that no financial support was received from any organization for the 


\section{Cureus}

submitted work. Financial relationships: All authors have declared that they have no financial relationships at present or within the previous three years with any organizations that might have an interest in the submitted work. Other relationships: All authors have declared that there are no other relationships or activities that could appear to have influenced the submitted work.

\section{References}

1. Bjekic M, Markovic M, Sipetic S: Clinical manifestations of primary syphilis. Braz J Infect Dis. 2012, 16:387389. 10.1016/j.bjid.2012.06.013

2. Neville BW, Damm DD, Allen CM, Chi A: History of syphilis. Oral and Maxillofacial Pathology. Elsevier, St. Louis; 2016. 5:164-190.

3. Romanowski B, Sutherland R, Fick GH, Mooney D, Love EJ: Serologic response to treatment of infectious syphilis. Ann Intern Med. 1991, 114:1005-1009. 10.7326/0003-4819-114-12-1005

4. Centers for Disease Control and Prevention. Sexually transmitted diseases surveillance: syphilis . (2016). Accessed: June 28, 2018: https://www.cdc.gov/std/stats16/Syphilis.htm.

5. Hook EW, Marra CM: Acquired syphilis in adults. N Engl J Med. 1992, 326:1060-1069. 10.1056/NEJM199204163261606

6. Rompalo AM, Lawlor J, Seaman P, Quinn TC, Zenilman JM, Hook EW 3rd: Modification of syphilitic genital ulcer manifestations by coexistent HIV infection. Sex Transm Dis. 2001, 28:448-454. 10.1097/00007435200108000-00004

7. CDC Morbidity Mortality Weekly Report. Transmission of primary and secondary syphilis by oral sexChicago 1998-2002. (2004). Accessed: June 28, 2018: https://www.cdc.gov/mmwr/preview/mmwrhtml/mm5341a2.htm.

8. Schroeter AL, Lucas JB, Price EV: Treatment for early syphilis and reactivity of serologic tests . JAMA. 1972, 221:471-476. 10.1001/jama.1972.03200180015003 